\title{
El trabajo fisioterapéutico y las etapas del duelo: Un enfoque interdisciplinario
}

\section{Physiotherapeutic work and the stages of grief: An interdisciplinary approach}

\section{Trabalho fisioterapêutico e os estágios do luto: Uma abordagem interdisciplinar}

Ebson Mauricio Briones-Arteaga ${ }^{\mathrm{I}}$

ebbrions70@hotmail.com

Oswaldo Robert Zambrano-Quinde II

oswaldozambra@hotmail.com

Recibido: 20 de diciembre de 2018 *Aceptacion: 24 de febrero de 2019 * Publicado: 05 de abril de 2019

${ }^{\text {I } M a ́ s t e r ~ e n ~ A c t i v i d a d ~ F i ́ s i c a ~ y ~ S a l u d, ~ L i c e n c i a d o ~ e n ~ l a ~ E s p e c i a l i z a c i o ́ n ~ F i s i o t e r a p i a, ~ T e c n o ́ l o g o ~ M e ́ d i c o ~}$ Especialidad Fisioterapia, Docente de la Universidad Laica Eloy Alfaro de Manabí, Manta, Ecuador.

${ }^{\text {II }}$ Magíster en Terapia Familiar Sistémica y de Pareja, Diploma Superior en Mediación, Especialista en Diseño Curricular por Competencias, Doctor en Psicología Clínica, Psicólogo Clínico, Docente de la Universidad Laica Eloy Alfaro de Manabí, Manta, Ecuador. 


\section{Resumen}

Las personas que acuden a fisioterapia no están exentas de situaciones de separación o pérdida de una persona, ideal, trabajo y/o amputación de un miembro. Todos estos acontecimientos de la vida generan inevitablemente un estado depresivo, dado que estos procesos psicológicos que entrañan perdida son, en esencia, muy parecidos. A pesar que, las respuestas a las pérdidas son peculiares y únicas, como cada ser humano, los patrones o estadios descritos en la conducta de las personas, de una u otra forma, son iguales para casi todos los casos. En este trabajo, se realizó un estudio del trabajo fisioterapéutico y las etapas del duelo concebidas a partir de un enfoque interdisciplinario, para ello se abordaron algunas teorías relacionadas al tema. Metodológicamente se siguió un método de tipo documental y bibliográfico a partir de la revisión de textos, documentales y la Web. Entre sus resultados, se pudo constatar que todos tenemos derecho a ser la persona que queremos ser y la única persona que puede quitarnos ese derecho somos nosotros mismos, asimismo en el caso particular de un periodo de amputación, probablemente una persona se sentirá muy estresado y es posible que experimente una pérdida de autoestima, de confianza en sí mismo, miedo o rechazo de su pareja, problemas financieros y que se cuestione su apariencia física.

Palabras clave: Trabajo fisioterapéutico; etapas del duelo; amputación y etapas del duelo.

\section{Abstract}

People who attend physiotherapy are not exempt from situations of separation or loss of a person, ideal, work and / or amputation of a member. All these life events inevitably generate a depressive state, since these psychological processes that involve loss are, in essence, very similar. Although, the responses to losses are peculiar and unique, like every human being, the patterns or stages described in the behavior of people, in one way or another, are the same for almost all cases. In this work, a study of the physiotherapeutic work and the stages of the duel conceived from an interdisciplinary approach was carried out, for which some theories related to the subject were approached. Methodologically, a method of documentary and bibliographic type was followed from the revision of texts, documentaries and the Web. Among its results, it was found that we all have the right to be 
the person we want to be and the only person who can take away that right is ourselves, also in the particular case of a period of amputation, probably a person will feel very stressed and you may experience a loss of self-esteem, self-confidence, fear or rejection from your partner, financial problems and your physical appearance being questioned.

Keywords: Physiotherapeutic work; stages of mourning; amputation and stages of mourning.

\section{Resumo}

As pessoas que frequentam a fisioterapia não estão isentas de situações de separação ou perda de uma pessoa, ideal, trabalho e / ou amputação de um membro. Todos esses eventos da vida inevitavelmente geram um estado depressivo, uma vez que esses processos psicológicos que envolvem a perda são, em essência, muito semelhantes. Embora as respostas às perdas sejam peculiares e únicas, como todo ser humano, os padrões ou etapas descritos no comportamento das pessoas, de uma forma ou de outra, são os mesmos para quase todos os casos. Neste trabalho, foi realizado um estudo do trabalho fisioterapêutico e das etapas do duelo concebido a partir de uma abordagem interdisciplinar, para o qual foram abordadas algumas teorias relacionadas ao assunto. Metodologicamente, um método de tipo documental e bibliográfico foi seguido a partir da revisão de textos, documentários e da web. Entre os resultados, verificou-se que todos têm o direito de ser a pessoa que você quer ser, e a única pessoa que pode tirar esse direito nós mesmos, também no caso de um período de amputação, provavelmente uma pessoa vai se sentir estressado e Você pode experimentar uma perda de autoestima, autoconfiança, medo ou rejeição de seu parceiro, problemas financeiros e sua aparência física sendo questionada.

Palavras-chave: Trabalho fisioterapêutico; fases de luto; amputação e fases de luto

\section{Introducción}

Etimológicamente, el término duelo tiene dos acepciones; la de "dolor" por una parte y la de "combate o desafío entre dos" por otra. A nivel intrapsíquico, el dolor surge por la pérdida del objeto y de las partes del yo proyectadas en el mismo. La capacidad para sentir dolor es fundamental para que el 
duelo se realice, sin dolor no hay duelo. El combate, también librado dentro del territorio intrapsíquico, consiste en una lucha entre la fantasía y la realidad, entre el amor y el odio, entre la vida y la muerte. Para que el duelo se resuelva satisfactoriamente, el juicio de realidad ha de imponerse a la fantasía, el amor tiene que triunfar sobre el odio de manera que la vida prevalezca sobre la muerte.

Este nuevo escenario es en el que la figura del Psicólogo cobra sentido en los Servicios Sociales en general y en los Servicios Sociales Comunitarios en particular. El psicólogo/a es el "experto" en comportamiento humano, de ahí su necesaria actuación en esta nueva concepción de intervención social que requiere de una incidencia sobre el comportamiento de los individuos y colectivos. La incorporación de los psicólogos a servicios sociales conlleva necesariamente una reflexión o análisis de las funciones que debe desempeñar este perfil, a qué problemáticas debe dirigir su atención y cuáles pueden ser los procedimientos más adecuados para emplear sus técnicas dentro del ámbito comunitario.

Teniendo en cuenta la multitud de problemas que se dan en entornos sociales tan cambiantes (aumento de la inmigración, envejecimiento de la población, problemáticas familiares, etc.) es preciso evaluar nuestros objetivos y actuaciones y adaptarnos a los cambios producidos en nuestra sociedad, evitando la cronificación del usuario en nuestros servicios y dando soluciones a problemas desde nuestra institución $\quad \mathrm{o}$ desde la derivación $\quad$ a otros dispositivos más especializado. Definiciones y consideraciones iniciales

\section{Desarrollo}

Para lograr entender el duelo, se hace necesario hacerse algunas preguntas sobre el estado de la cuestión ¿Qué es el duelo?, ¿Cómo y cuándo intervenir?, ¿Estamos dando categoría de enfermedad a un proceso natural? ¿Qué cambios culturales se están produciendo y cómo están afectando al duelo? ¿Qué dice la investigación al respecto?

Si bien su respuesta no puede ser inmediata ni inequívoca, empezaremos por decir que todos nos enfrentamos en algún momento de nuestras vidas a la muerte, y cuando hablamos de esta podemos hacer referencia a tres dimensiones: 
- Muerte biológica: parada de las funciones de nuestro organismo. Hay un cese del latir del corazón o de la actividad cerebral.

- Muerte psicológica (Bayés, 2001): conocimiento subjetivamente cierto que suscita en un momento concreto de la vida de que "voy a morir".

- Muerte socio-cultural: que hace referencia al conjunto de rituales de luto y duelo que se realizan alrededor de la muerte de una persona en un contexto social y cultural determinado.

Por otra parte las situaciones de pérdida a las que nos podemos enfrentar también son diversas, y según Tizón (2004) pueden ser de cuatro tipos: materiales (posesiones, herencias, objetos), evolutivas (infancia, adolescencia, menopausia y andropausia y jubilación), relacionales (de seres queridos, separaciones, abandonos) e intrapersonales (pérdidas significativas como desengaños, corporales, capacidades).

En general una pérdida supone experimentar momentos difíciles de la vida de una persona, a la que en la mayoría de los casos nos vamos adaptando para seguir viviendo. En particular la pérdida relacional por muerte de un ser querido suele ser una situación especialmente dura, por la que tarde o temprano todos vamos a pasar. No obstante, a pesar de ser un proceso natural del ciclo de la vida, sufriremos una serie de reacciones, en principio normales, que es lo que vamos a denominar duelo.

El término duelo etimológicamente, proviene del latín dolus (dolor) y han sido muchos los autores que lo han definido. Así, Freud en su trabajo Duelo y Melancolía (1915), lo definió como "una reacción a la pérdida de un ser amado o de una abstracción equivalente". Bowlby (1993) define el duelo como "todos aquellos procesos psicológicos, conscientes e inconscientes, que la pérdida de una persona amada pone en marcha, cualquiera que sea el resultado".

Según la Real Academia Española (2001), el concepto duelo tiene varios significados: 
- Dolor, lástima, aflicción o sentimiento.

- Demostraciones que se hacen para manifestar el sentimiento que se tiene por la muerte de alguien.

- Reunión de parientes, amigos o invitados que asisten a la casa mortuoria, a la conducción del cadáver al cementerio o a los funerales.

El duelo como proceso se define como "un conjunto de procesos psicológicos y psicosociales que siguen a la pérdida de una persona con la que el sujeto en deudo estaba psicosocialmente vinculado" (Tizón, 2004).

En el proceso de duelo normal tradicionalmente se establecían una serie de etapas por las cuales el doliente tenía que pasar para la recuperación y superación del mismo, en el proceso del duelo por parte del doliente al establecerlo por etapas. La visión del duelo que presentan estos autores es más activa y prefieren hablar de tareas que el doliente tiene que realizar y superar sin necesidad de seguir un orden determinado. De esta forma el duelo tiene más en cuenta las diferencias individuales, que pueden variar en función de las características personales, la naturaleza de la relación con lo perdido o el contexto en donde se produce la pérdida.

En esta línea nos encontramos con las facetas de afrontamiento activo propuestas por Attig (1996) y las tareas de William Worden (1997), siendo este último uno de los autores más reconocidos y aceptados por su modelo de "tareas" que el doliente tiene que elaborar tras la pérdida:

Tarea I: Aceptar la realidad de la pérdida

Tarea II: Trabajar las emociones y el dolor de la pérdida

Tarea III: Adaptarse a un medio en el que el fallecido está ausente

Tarea IV: Recolocar emocionalmente al fallecido y continuar viviendo.

Cabe exponer algunos conceptos básicos que se deben considerar durante el trabajo fisioterapéutico: 
Negación. La negación la suelen experimentar personas que se someten a amputaciones por traumatismo; normalmente aquéllas a las que se les ha practicado amputaciones quirúrgicas no la sufren.

Rabia. A menudo se culpa de la pérdida a Dios, al médico o a otras personas.

Regateo. En esta fase, los pacientes intentan posponer la realidad de la amputación y la mayoría intentará regatear con su doctor o alguna autoridad superior como, por ejemplo, una figura religiosa.

Depresión. En esta etapa, la depresión sustituye a la rabia. Ésta es probablemente la fase más complicada de la adaptación, pero también desaparecerá. No es una depresión clínica; es normal. Los síntomas más frecuentes son el exceso o la falta de sueño, los sentimientos negativos sobre la realidad y el futuro, los sentimientos de desesperación y hablar de la muerte. La depresión no es un signo de debilidad; sin embargo, no debería sentirse así. Es tratable y usted no debería dudar en pedir ayuda a su médico, enfermera, familiares y amigos.

Aceptación y esperanza. Con el tiempo, aceptará la pérdida y volverá a su hacer su vida normal. Esto se consigue más fácilmente si habla con alguien que haya superado todo el proceso y le pueda dar algunos consejos que le ayuden a afrontar la pérdida.

Tristeza complicada. Aunque no es común en pacientes amputados, usted debe ser consciente de sus síntomas, que incluyen aislamiento severo, comportamiento violento, ideas suicidas, adicción al trabajo, depresión severa o prolongada, pesadillas y evitar pensar en su amputación. Si experimenta alguno de estos síntomas, por favor, informe a los correspondientes profesionales médicos.

Múltiples factores. Además de la pérdida de un miembro, puede que experimente otras complicaciones, dependiendo de la situación y del apoyo que reciba de otras personas.

En atención a las exposiciones anteriores, se destaca la posición de Kübbler-Ross (2004), quien explica que existen etapas en el proceso de duelo, y en los casos que se demandan en fisioterapia no hay recetas y esta no es la excepción, dependerá de cada paciente y de sus propias herramientas: En la actualidad el ser humano le da una valoración muy importante al cuerpo, los estándares 
estéticos, sociales y psicológicos establecen la necesidad de que el cuerpo de las personas estén integrados al $100 \%$. La pérdida de una parte del cuerpo supone la pérdida de funcionalidad dentro del medio social, supone pérdida estética, pérdida de oportunidades, educativas y laborales. Casi todo está concebido y diseñado para el ser humano completo.

Las amputaciones pueden ocurrir en tres formas básicas, amputaciones en el nacimiento congénitas, amputaciones accidentales o traumáticas, que ocurren regularmente en accidentes de tránsito y accidentes laborales y amputaciones por enfermedad. Cada una de ellas trae consecuencias psicológicas diferentes.

Las amputaciones de nacimiento traen consigo problemas para el desarrollo psicológico cognitivo y social, sin embargo en este caso las consecuencias psicológicas serán en primer instancia para los padres y el grupo familiar del niño que nace con una atrofia corporal en alguna de sus extremidades. Las repercusiones psicológicas en el menor se van apareciendo conforme el niño va teniendo consciencia de su cuerpo y del apoyo que tenga de sus padres y familiares va a depender si existen consecuencias negativas o no, generalmente este tipo de personas, se va adaptando a su situación y va a estar mejor adaptado que las personas que sufren de una amputación súbita.

Amputaciones por enfermedad, estas suceden en la mayoría de los casos en las personas de la tercera edad con complicaciones por enfermedad crónico-degenerativas como la diabetes y el cáncer y la amputación es anticipada al paciente y regularmente tiene el objetivo de evitar complicaciones mayores para la salud, mejorar la calidad de vida y en todo caso evitar la muerte y el proceso de adaptación es menos complicado que en las amputaciones traumáticas.

Amputaciones súbitas o amputaciones traumáticas, estas ocurren, valga la redundancia, en un hecho traumático, en un accidente automovilístico, en un accidente laboral, en un atentado violento, en un ataque terrorista. En estos casos las consecuencias psicológicas tienen mayores complicaciones dependiendo de las características de cada persona, de su edad, su sexo, su estado de desarrollo, su actividad laboral o profesional. 
Puede tener mayores afectaciones psicológicas si ocurre en una mujer que en un hombre debido a las mayores exigencias estéticas en la imagen femenina, las mismas consecuencias de naturaleza estética, pueden influir si ocurre durante la etapa de la adolescencia, cuando el joven va cimentando su desarrollo personal en sus características personales. Una amputación de una pierna puede tener mayor afectación psicológica en un deportista que en un profesionista. Las consecuencias psicológicas de una amputación traumática van a variar dependiendo de las características de la personalidad del amputado.

Sí, somos un poco diferentes, pero todo el mundo lo es, así podemos entenderlo a partir de este argumento; después de mi amputación, me sentaba en casa sin querer salir porque me preocupaba demasiado mi cuerpo. Tenemos una piscina y me daba vergüenza incluso usarla. Caray, jeso sí era una tontería! No caiga en la misma trampa. Esta falsa vergüenza está en su mente. Nos preocupados porque nos da miedo lo que piensen otros de nuestra apariencia. Me sentí así hasta que una noche estaba viendo el programa America's Funniest Home Videos (Los vídeos caseros más divertidos) y vi la cosa más extraña que he visto nunca. Era un perro corriendo a toda velocidad con dos amputaciones, ¡ambas en el mismo costado! Pensé que era extraño, ¿Pero usted cree que el perro pensaba lo mismo? Claro que no. El perro era capaz de afrontar su pérdida, y ahí estaba en el patio jugando. A él no le importaba ser diferente, lo único que le importaba era disfrutar la vida, pues podemos aprender mucho de un perro.

Si tiene estas falsas impresiones sobre sí mismo, trate de superarlas. Hay muchas fuentes de ayuda disponibles y usted no debería tener miedo de hablar de esos problemas con alguien. La mayoría de las personas con amputaciones han pasado por lo mismo, pero las superaron, y usted también lo hará.

Soy un ferviente defensor del asesoramiento entre pacientes amputados, y si usted tiene la suerte de recibir la visita de un compañero asesor, éste se tomará su tiempo para sentarse con usted a solas y escuchar sus preocupaciones. También se tomará su tiempo para hablar con su familia y su pareja. Mientras usted supera este proceso de adaptación, sea consciente de que toda su familia está pasando por lo mismo. Es importante sacarse esos sentimientos y comunicarse con sinceridad. De otro modo, esos sentimientos se enconarán y le causarán más daño a usted, a su familia y a su pareja. 


\section{Metodología}

El estudio siguió una metodología de tipo documental y bibliográfico, que según Hernández y col (2014), refiere a la consulta de libros, material impreso, información Web y otros medios bibliográficos de referencia relacionados con un tema o una variable de estudio de una investigación. Los resultados se obtuvieron considerando el análisis hermenéutico, posterior a la lectura y comprensión de lo consultado.

\section{Conclusiones}

Durante un periodo de amputación, probablemente una persona se sentirá muy estresada. Es posible que experimente una pérdida de autoestima, de confianza en sí mismo, miedo o rechazo de su pareja, problemas financieros y que se cuestione su apariencia física. Probablemente, uno de los problemas más difíciles es perder el sentimiento de independencia y tener que depender de otros hasta en las necesidades más básicas.

La pérdida de confianza y de autoestima, por ejemplo, no es necesaria. La confianza en sí mismo y la autoestima están en la mente, no en la pierna o el brazo que ha perdido. Sigue siendo la persona valiosa que era antes. Con el tiempo, se percatará de que aprenderá a adaptarse y su actitud cambiará para bien. Otro error que se comete tras una amputación es la forma en que percibí su cuerpo. Después de una amputación, es muy normal creer que ya no resulta atractivo a su pareja. Tal vez pueda ser cierto en algunos casos, pero si la pareja realmente le ama, la pérdida de un miembro no supondrá ninguna diferencia en su relación, sexual o de otro tipo. Por supuesto, serán necesarios algunos ajustes, que pueden hacerse y se harán, y las cosas, con el tiempo, volverán a la normalidad.

Todos tenemos derecho a ser la persona que queremos ser y la única persona que puede quitarnos ese derecho somos nosotros mismos. Para ello se aconseja a muchos pacientes con amputaciones, de todas las dificultades que pasarán. Argumentándoles, que deben ver la vida de manera positiva para asegurarse un futuro productivo. 
Es muy fácil caer en el abismo de la autocompasión, decidir que no vale la pena y pasar parte o el resto de su valiosa vida hibernando. No deje que eso ocurra. Si necesita ayuda, hay muchos recursos que pueden serle útiles. Si se tiene un compañero que le asesora háblelo con él. Después de todo, ha pasado por lo mismo que usted está experimentando ahora, por lo tanto también puede.

Virtualmente toda persona que ha sufrido una amputación afirma experimentar sensaciones que parecen emanar de la parte amputada del miembro. La mayoría de las veces estas sensaciones fantasma son indoloras y de intensidad lo suficientemente baja como para no provocar más que una moderada perturbación.

Las sensaciones son generalmente similares a aquellas que se sienten en un miembro intacto, incluyendo calor, picazón, sensación de posición y una moderada compresión.

El conocimiento de los detalles de la forma del miembro y la percepción de la capacidad de moverlo tienden a debilitarse con el tiempo. No obstante, casi todos los amputados afirman que continúan sintiendo al menos algunas sensaciones fantasma lo largo de toda su vida. Cuando las sensaciones fantasmas se vuelven para el amputado lo suficientemente intensas como para definirla como un dolor se las denomina dolencia fantasma.

\section{Referencias Bibliográficas}

Attig, T. (1996). La importancia de concebir la pena como un proceso activo, Death Studies, 15 (4), 385-393, DOI: 10.1080 / 07481189108252443

Bayés, R. (2001): Psicología del sufrimiento y de la muerte. Ediciones Martínez Roca, Barcelona.

Bowlby, J (1993). Charles Darwin: A new biography. London: Hutchinson

Freud, S. (1915). Duelo y melancolía. Obras Completas de Sigmund Freud. Vol. XIV: Amorrortu Editores, Buenos Aires.

Hernandezi, R., Fernández, C., \& Bautista, P. (2014). Metodología de la Investigación. México: Mcgraw-hill/Interamericana Editores. S.A. DE C.V. 
El trabajo fisioterapéutico y las etapas del duelo: Un enfoque interdisciplinario

Kübbler-Ross, E. (2004): La rueda de la vida. Ediciones B. S.A., Barcelona.

Real Academia Española (2001). Recuperado de:

http://www.rae.es/recursos/diccionarios/diccionarios-anteriores-1726-2001/diccionario-de-

la-lengua-espanola

Tizón, J. (2004). El duelo en atención primaria a la salud (I). Formación Médica Continuada en Atención Primaria. 3 (2a), 547-66. Recuperado de: https://www.casadellibro.com/libroperdida-pena-duelo-vivencias-investigacion-y-asistencia/9788449316

William Worden. (1997). El tratamiento del duelo: asesoramiento psicológico y terapia. Ediciones Paidós Ibérica, Barcelona. 